\title{
Avascular Necrosis of Femur in Case of Severe Aplastic Anemia: A Case Report
}

\author{
Himani Dhingra ${ }^{1}$, Geeta Mandhani ${ }^{2}$, Manas Kalra $^{3}$, Amita Mahajan $^{4}$
}

\section{Abstract}

Outcomes of severe aplastic anemia (SAA) have drastically improved over the last decade with most patients being cured after an appropriate therapy. Long-term side effects are hence becoming increasingly important. Avascular necrosis (AVN) is one of such debilitating complications. We hereby report the unusual association of AVN of hip in two pediatric patients who were treated with immunosuppressive therapy (IST) with short course of low-dose steroids and discuss the existing literature.

Keywords: Aplastic anemia, Avascular necrosis, Immunosuppressive therapy.

Journal of Orthopedics and Joint Surgery (2019): 10.5005/jp-journals-10079-1004
\end{abstract}

\section{INTRODUCTION}

Avascular necrosis (AVN) is a pathological condition resulting in cellular death of bone components due to interruption of vascular supply. Avascular necrosis usually affects the epiphysis of long bones; however, small bones with single terminal blood supply can also be involved. Risk factors that predispose to AVN can be traumatic and nontraumatic. ${ }^{1}$ Nontraumatic AVN is largely idiopathic; however, it can be secondary to the use of corticosteroids, hypercoagulability, hyperlipidemia, and bone marrow transplant (BMT). ${ }^{2}$ The severity of clinical presentation may range from asymptomatic to a debilitating painful condition with a reduction in joint mobility and degenerative changes. Magnetic resonance imaging (MRI) is the most sensitive and specific imaging modality for diagnosis. ${ }^{3}$ It is also helpful for grading of disease severity and screening of contralateral hip. The treatment options can vary depending on age, site, and severity of involvement. Treatment outcomes correlate directly with disease severity.

Though AVN has been observed in patients with aplastic anemia, a causal relationship has not yet been determined. The majority of cases that have been reported previously are following allogeneic $\mathrm{BMT}^{4}$ or immunosuppressive therapy (IST) with antithymocyte globulin (ATG), attributed mainly to the use of high dose methylprednisolone (MPS). ${ }^{5}$ However, it has been infrequently reported with ATG and the current steroid regimen. This case report highlights the development of AVN of head of femur in two young males with severe aplastic anemia (SAA) following IST and short course, low-dose MPS for the prevention of serum sickness.

\section{Case Description}

\section{Case 1}

A 17-year-old male patient, with SAA, presented to our hospital for further management. Due to logistic constraints allogeneic BMT could not be offered as frontline therapy. His initial treatment was comprised of IST with ATG along with short course of low-dose steroids (intravenous MPS $1 \mathrm{mg} / \mathrm{kg} /$ day on days 1-4 followed by oral prednisolone $1 \mathrm{mg} / \mathrm{kg} /$ day for 10 days then tapered over a week) and cyclosporine A.

After 2 years, he presented with progressively increasing pain over both hip joints with limp while walking. On examination, \begin{tabular}{l}
\hline${ }^{1-4}$ Department of Pediatric Hematoncology, Indraprastha Apollo \\
Hospitals, New Delhi, India \\
Corresponding Author: Himani Dhingra, Department of Pediatric \\
Hematoncology, Indraprastha Apollo Hospitals, New Delhi, India, \\
Phone: +91 7015072317, e-mail: drhimani458@gmail.com \\
How to cite this article: Dhingra H, Mandhani G, Kalra M, et al. \\
Avascular Necrosis of Femur in Case of Severe Aplastic Anemia: A Case \\
Report. J Orth Joint Surg 2019;1(1):38-40. \\
Source of support: Nil \\
Conflict of interest: None
\end{tabular}

there was tenderness present over both hips with restricted range of motion and shortening of left lower limb by $1.5-\mathrm{cm}$. X-ray pelvis and bilateral hip (Fig. 1) done showed amodulation of left femoral head. Radiolucent defects with surrounding sclerosis were seen in both femoral heads with narrowing of bilateral hip joint spaces. Magnetic resonance image pelvis (Figs 2 and 3) showed circumscribed geographic subchondral lesions anterosuperiorly in both femoral heads, appearing hypointense on T1WI. Bilateral femoral heads showed articular surface irregularity along with focal

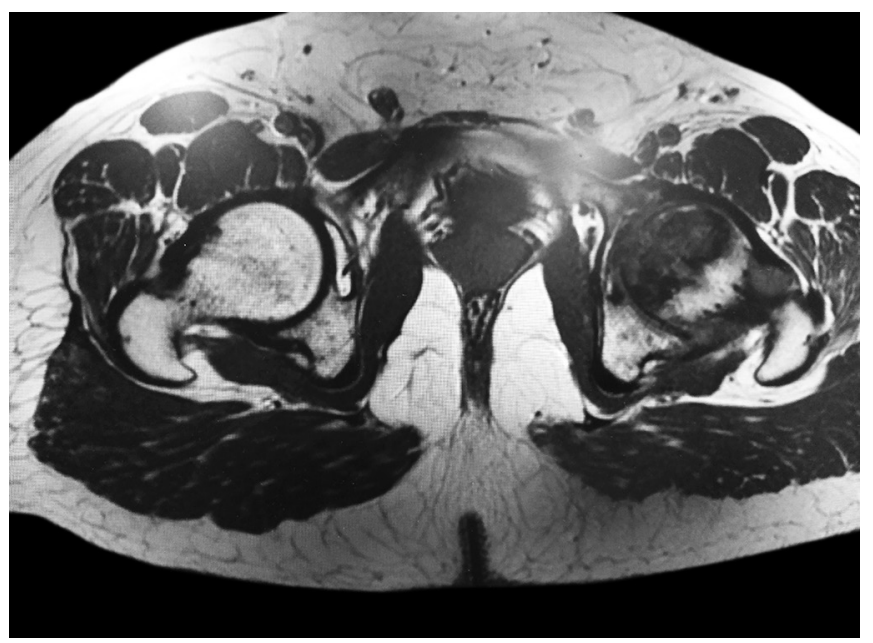

Fig. 1: Avascular necrosis of both femoral heads (left $>$ right) with arthritis of hip joints 


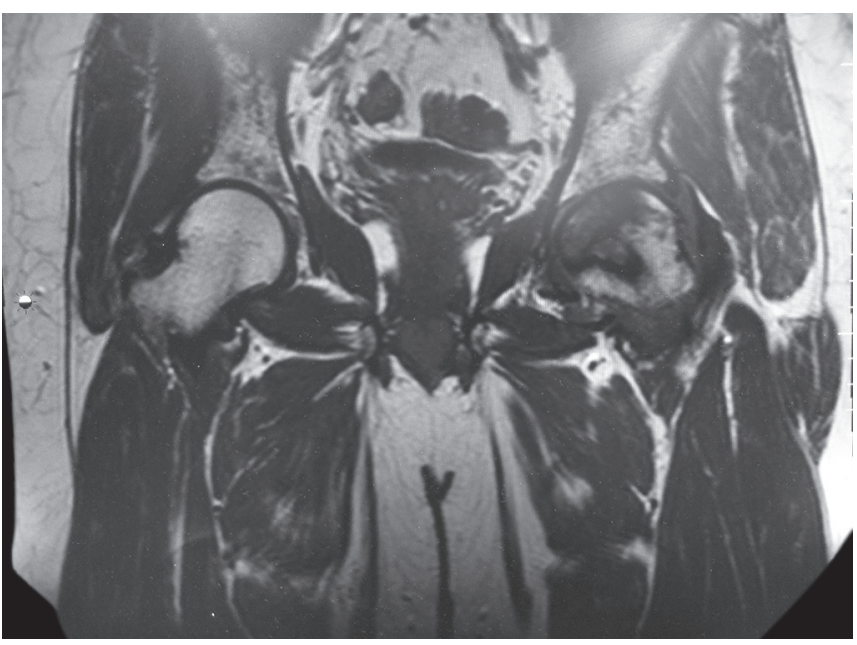

Fig. 2: Bilateral articular surface irregularity with focal subchondral defects (left $>$ right)

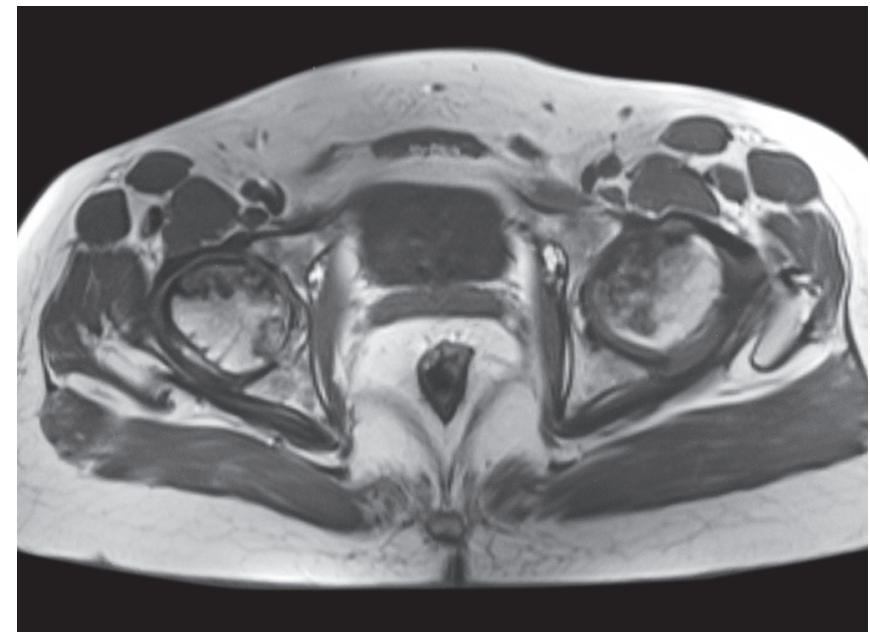

Fig. 4: Area of altered signal intensity in subchondral location of head of left femur

subchondral defects; however, this was more marked on left side in anterosuperior aspect. This was consistent with AVN of bilateral femoral head (left grade IV, right grade III) with arthritis of hip joints. Bilateral joint spaces were reduced. He underwent a total left hip arthroplasty after failure of conservative measures. However, his primary disease condition subsequently relapsed and he underwent an allogeneic BMT.

\section{CASE 2}

A 16-year-old male patient, diagnosed with SAA was also treated with IST with ATG along with cyclosporine and short course of low-dose steroids. 2 years following the treatment, he developed complaints of severe pain in left hip with painful abduction and internal rotation. Magnetic resonance image pelvis with both hip (Figs 4 and 5) showed serpiginous area of low signal intensity in subchondral region of head of left femur consistent with AVN (grade II). Associated short T1 inversion recovery (STIR) hyperintensity suggestive of marrow edema was seen extending into neck and intertrochanteric region of femur. There was no evidence of flattening or collapse of head of femur. After failure of conservative

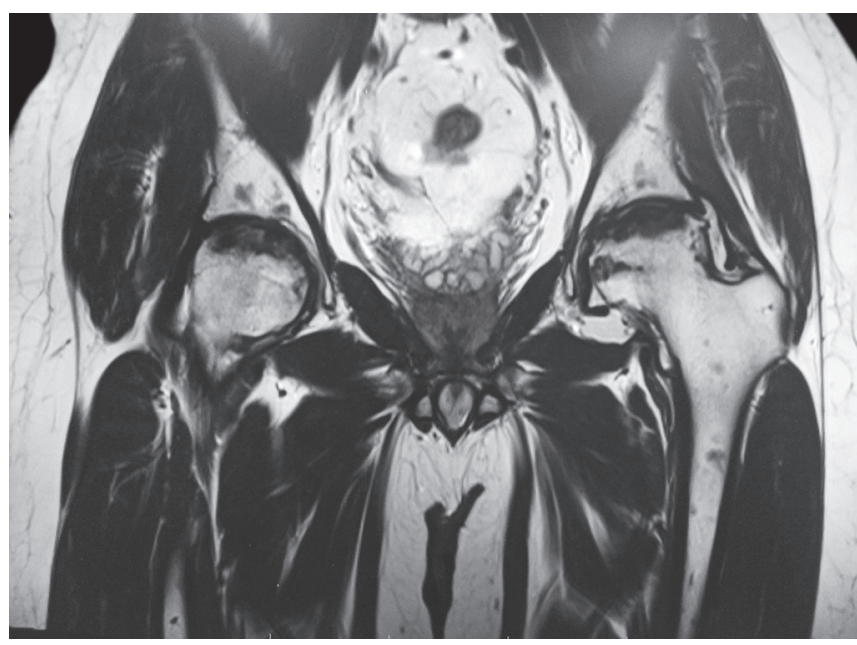

Fig. 3: Subchondral lesions with altered signal intensity in both femoral heads

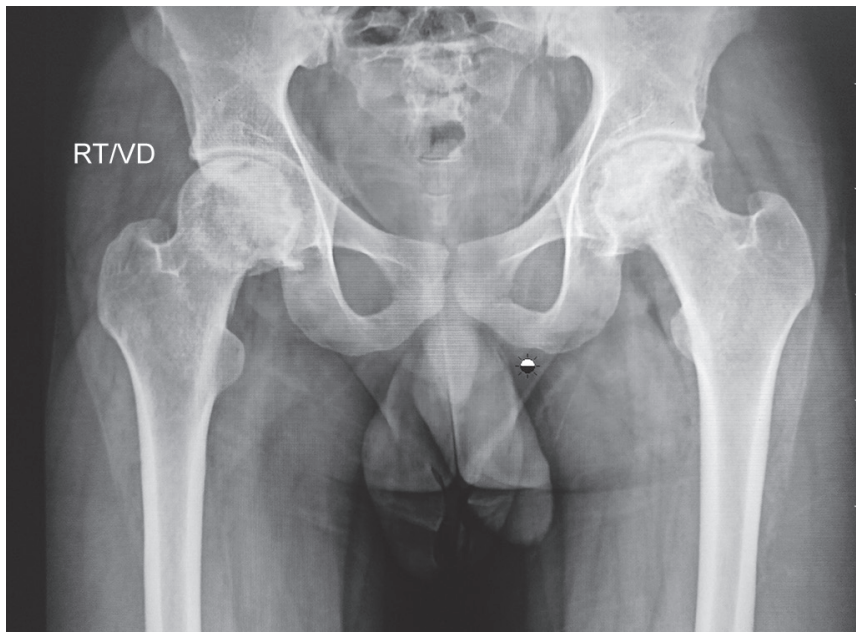

Fig. 5: Avascular necrosis of left hip

management, he underwent core decompression of left femoral head to decrease intramedullary pressure, improve ischemia, and prevent further joint destruction. Postprocedure patient was symptomatically better and pain subsided. A reassessment on MRI pelvis was done 4 months postsurgery, regression of edema of head, neck, and intertrochanteric region of femur.

Both the patients received IST and short course of low-dose steroids as a part of management of SAA. Both of them developed AVN of head of femur almost 2 years following IST. Currently, their primary disease is in remission and both the patients are ambulatory without support.

\section{Discussion}

Aplastic anemia is a potentially severe hematological disorder characterized by hypocellular fatty marrow resulting into cytopenias. With current advances in therapeutic options, more than $90 \%$ patients ${ }^{6}$ can be cured, and hence, long-term complications are becoming increasingly important.

Immunomediated destruction of hematopoietic cells is one of the main pathophysiologic mechanisms of acquired aplastic anemia. Hence, IST continues to be the treatment of choice in 
Table 1: Characteristics of patients with aplastic anemia that developed avascular necrosis

\begin{tabular}{|c|c|c|c|c|c|c|c|}
\hline S. no. & Study & Age at diagnosis & $\begin{array}{l}\text { Treatment given for } \\
\text { aplastic anemia }\end{array}$ & Incidence of AVN & $\begin{array}{l}\text { Median time } \\
\text { following treatment }\end{array}$ & $\begin{array}{l}\text { Unilateral or } \\
\text { bilateral }\end{array}$ & Management \\
\hline \multirow[t]{3}{*}{1} & Marsh et al. ${ }^{5}$ & & $\begin{array}{l}\text { ALG followed by } \\
\text { high-dose MPS }\end{array}$ & $21 \%$ & 14 months & $6 / 49$ unilateral & $\begin{array}{l}5 \text { surgical } \\
\text { intervention }\end{array}$ \\
\hline & & & $\begin{array}{l}\text { ALG followed by short } \\
\text { course of low-dose } \\
\text { prednisolone }\end{array}$ & No & & 5/49 bilateral & $\begin{array}{l}3 \text { total hip } \\
\text { replacement }\end{array}$ \\
\hline & & & 5 patients, no treatment & & & $\begin{array}{l}2 / 49 \text { more } \\
\text { than } 2 \text { joints }\end{array}$ & \\
\hline \multirow[t]{3}{*}{2} & Park et al. ${ }^{7}$ & 19-46 years & 12 patients, ALG & $7.9 \%$ & 6 months -4 years & 14 bilateral & \\
\hline & & & $\begin{array}{l}7 \text { patients, prednisolone } \\
(4 \mathrm{mg} / \mathrm{kg} \text { tapered over } \\
3 \text { weeks })\end{array}$ & & & 5 unilateral & \\
\hline & & & IST & & & & \\
\hline 3 & Plichta et al. ${ }^{8}$ & 19 years & & & & Unilateral & Conservative \\
\hline
\end{tabular}

patients with SAA, who do not have a matched donor. ${ }^{6} \mathrm{~A}$ short course of low-dose steroids is usually administered with IST to reduce the risk of serum sickness. ${ }^{6}$

The commonly presumed pathological mechanism for AVN in SAA is a compromised blood circulation of the bone, leading to cell death. During revascularization, osteoclasts cause bone resorption resulting in demineralization and trabecular thinning, and subsequently mechanical failure.

The majority of cases of AVN with aplastic anemia are reported with treatment either after $\mathrm{BMT}^{4}$ or with the use of high-dose MPS with ATG. In a study by Marsh et al., ${ }^{5}$ the incidence of AVN in patients treated with antilymphocyte globulin (ALG) followed by high-dose MPS was 14\%; however, there were no cases of AVN in a historical group of 61 patients with aplastic anemia treated with an identical regimen of ALG but using a short course of low-dose prednisolone. ${ }^{5}$ In this study, the authors attributed AVN to corticosteroids administered at high doses. The mechanism postulated was increased pressure and fat in the marrow cavity leading to a collapse of the sinusoids in the femoral head, resulting in ischemia.

The cumulative incidence of AVN in aplastic anemia reported by Park et al. on 241 patients was $7.9 \% .^{7}$ In this study, AVN was diagnosed in five patients with aplastic anemia before any medication was administered, suggesting that aplastic anemia itself can be an etiology of AVN. The authors proposed that in the fatty marrow of aplastic anemia, the sinusoidal system is replaced by capillaries that are sparse. The perfusion of the marrow decreases gradually, and consequent vascularization of this marrow is ineffective due to venous stasis. As a result of this characteristic vascular structure, fatty marrow is more vulnerable to ischemia than hematopoietic marrow.

Overall, the pathophysiology of AVN in SAA remains unclear. It is probable that the disease process, treatment, and genetic factors all play a role. Our patients had received low-dose steroids in addition to ATG as a part of IST. No long-term steroids were given, highlighting the fact that either aplastic anemia itself or low-dose steroid acted as a precipitating factor for AVN in a background of compromised bone vascularity. Table 1 summarizes the existing literature on the characteristics of patients with aplastic anemia that developed AVN.

\section{Conclusion}

Avascular necrosis is a significant long-term complication that can compromise joint functionality and quality of life in afflicted survivors of aplastic anemia. Preservation of joint function and control of pain are important factors in management of these patients. Early diagnosis and management may help in minimizing the physical and emotional impact of this complication. More research needs to be done to delineate the etiological and genetic factors to identify high risk population.

\section{References}

1. Shah KN, Racine J, Jones LC, et al. Pathophysiology and risk factors for osteonecrosis. Curr Rev Musculoskelet Med 2015;8(3):201-209. DOI: 10.1007/s12178-015-9277-8.

2. Fink JC, Leisenring WM, Sullivan KM, et al. Avascular necrosis following bone marrow transplantation: a case-control study. Bone 1998;22(1):67-71. DOI: 10.1016/S8756-3282(97)00219-6.

3. Murphey MD, Roberts CC, Bencardino JT, et al. ACR appropriateness criteria osteonecrosis of the hip. J Am Coll Radiol 2016;13(2):147-155. DOI: 10.1016/j.jacr.2015.10.033.

4. Konopacki J, Porcher R, Robin M, et al. Long-term follow up after allogeneic stem cell transplantation in patients with severe aplastic anemia after cyclophosphamide plus antithymocyte globulin conditioning. Haematologica 2012;97(5):710-716. DOI: 10.3324/ haematol.2011.050096.

5. Marsh JC, Zomas A, Hows JM, et al. Avascular necrosis after treatment of aplastic anemia with antilymphocyte globulin and high-dose methylprednisolone. Br J Haematol 1993;84(4):731-735. DOI: 10.1111/j.1365-2141.1993.tb03153.x.

6. Hartung DH, Olson ST, Bessler M. Acquired aplastic anaemia in children. Pediatric Clin North Am 2013;60(6):1311-1336. DOI: 10.1016/ j.pcl.2013.08.011.

7. Park J, Jun J, Kim Y, et al. Osteonecrosis of the hip in patients with aplastic anemia. J Korean Med Sci 2002;17(6):806-810. DOI: 10.3346/ jkms.2002.17.6.806.

8. Plichta P, Pawelec K. Avascular necrosis of the femoral head in a patient with severe aplastic anaemia. Med Wieku Rozwoi 2011;15(1):101-105. 